\title{
How to Reuse a Faceted Classification and Put It on the Semantic Web
}

\author{
Bene Rodriguez-Castro, Hugh Glaser, and Leslie Carr \\ School of Electronics and Computer Science, University of Southampton, \\ Southampton SO17 1BJ, UK \\ \{b.rodriguez, hg, lac\}@ecs.soton.ac.uk \\ http://www.ecs.soton.ac.uk
}

\begin{abstract}
There are ontology domain concepts that can be represented according to multiple alternative classification criteria. Current ontology modeling guidelines do not explicitly consider this aspect in the representation of such concepts. To assist with this issue, we examined a domain-specific simplified model for facet analysis used in Library Science. This model produces a Faceted Classification Scheme (FCS) which accounts for the multiple alternative classification criteria of the domain concept under scrutiny. A comparative analysis between a FCS and the Normalisation Ontology Design Pattern (ODP) indicates the existence of key similarities between the elements in the generic structure of both knowledge representation models. As a result, a mapping is identified that allows to transform a FCS into an OWL DL ontology applying the Normalisation ODP. Our contribution is illustrated with an existing FCS example in the domain of "Dishwashing Detergent" that benefits from the outcome of this study.
\end{abstract}

Keywords: facet analysis, faceted classification, normalisation, ontology design pattern, ontology modeling.

\section{Introduction}

Ontologies remain as one of the key components needed for the realization of the Semantic Web vision. They bring with them a broad range of development activities that can be grouped into what it is referred to as Ontology Engineering. Ontology Engineering for the Semantic Web is a very active research area and has experienced remarkable advancements in recent years, although it is still relatively new compared to other engineering practices within Computer Science or other fields. A constant ongoing effort in Ontology Engineering deals with harnessing the field with sound development methodologies analogous to those successfully employed in Software Engineering for decades. One of the objectives of these methodologies is to address areas of the ontology development process vulnerable to ad-hoc practices that could potentially lead to unexpected or undesirable results in ontology artifacts.

This paper describes a specific, very recurrent modeling scenario in ontology development, subject to such vulnerability. The scenario consists of domain-specific 
concepts that can be represented according to multiple alternative classification criteria. To the best of our knowledge, guidelines for the conceptualization and representation of domain-specific concepts prone to be described based on multiple (potentially alternative) classification criteria, has not been explicitly considered in the context of ontology modeling for the Semantic Web.

General examples of domain-specific concepts that exhibit the characteristics described abound, going from a "bibliographic reference", (which could be classified according to several criteria such as "subject", "author", "publication venue", etc.); to a "toy" (which could be classified based on "suitable age", "brand", "subject type", etc.). The list of examples can go on. We have seen in our own experience that lack of specific design guidelines leaves ample room for conceptual errors when trying to develop a simple domain-specific ontology model for such concepts. For example, common mistakes when trying to represent these concepts and their classification criteria are to use subsumption relations between classes when in fact a part-of relation would be in order, or to use subsumption to model relationships that are outside OWL DL expressivity altogether.

Other examples of domain-specific concepts that can fit into the modeling scenario described are particularly interesting because they are used in wellknown ontology development literature using OWL. They include: "Wine" [1], "Person" (in the context of family history relations) [2], or "Pizza" 3]. However, in none of them, they refer explicitly to the various classification criteria of the domain concept that are considered implicitly, nor attempt to represent these criteria explicitly in the respective ontology models developed.

To assist with these issues, we aim to put forward an initial set of basic design guidelines to mitigate the opportunity for ad-hoc modeling decisions in the development of ontologies for the problem scenario described. To obtain the conceptual model of a domain-specific concept and its multiple classification criteria we examined a simplified model for facet analysis in the field of Library and Information Science 44. The outcome of this facet analysis is a Faceted Classification Scheme (FCS) for the domain concept in question where in most cases a facet would correspond to a classification criterion. To obtain an ontology representation of the FCS, we examined the Normalisation Ontology Design Pattern (ODP) [5] 6] [7. A comparative analysis between a FCS and the Normalisation ODP revealed the existence of key similarities between both knowledge representation paradigms. The similarities allowed us to identify a series of mappings to transform a FCS into an OWL ontology applying the Normalisation pattern. Moreover, the ontology model obtained through this process contains a valid OWL DL representation of the classification criteria involved in the characterization of the domain concept.

To illustrate our contribution, we used throughout the document an existing FCS example in the domain of "Dishwashing Detergent" 8]. In fact, there are aspects of the work presented in this paper that could be viewed as a follow-up to [8] in the context of the Semantic Web and we attempted to acknowledge that in our title. 
There is an additional important use case worth highlighting for motivating the need of this work as well. That is the modeling of the concept "Fault" in the domain of resilient and dependable computer systems. The representation of "Fault" is part of an ontology featured in a web portal knowledge base (RKBExplore1 1 ) for the project ReSIST ${ }^{2}$ (Resiliance for Survivability in Information Society Technologies) 9.

The rest of this paper is structure as follows: Section 2 describes the structure and elements of a generic FCS; Section 3 does likewise regarding the Normalisation ODP; Section 4 introduces the alignments identified between both knowledge representation paradigms to enable the transformation of a generic FCS into a normalised ontology; Section 5 provides a comparison to previous work closely related to our proposal; and finally, Section 6 concludes the paper with some final remarks.

\section{Faceted Classification Scheme}

This section remarks the main features of a FCS involved in the comparative analysis to the Normalisation ODP for a given domain of discourse, while a thorough overview of facet analysis and FCSs can be found in [4] 10]. The latter also explores how FCSs compare to other knowledge representation approaches in classification and provides an account of its strengths and limitations.

Denton [8] $(\S 0)$ characterized a FCS for a given domain as follows: "a set of mutually exclusive and jointly exhaustive categories, each made by isolating one perspective on the items (a facet), that combine to completely describe all the objects in question, and which users can use, by searching and browsing, to find what they need".

However, in order to develop a FCS it is required to go through the process of Facet Analysis. Vickery [8] (§ 2.3) describes Facet Analysis as: "The essence of facet analysis is the sorting of terms in a given field of knowledge into homogeneous, mutually exclusive facets, each derived from the parent universe by a single characteristic of division".

The key to Facet Analysis and FCSs is the notion of facet. Spiteri [4] simplified existing principles used in established Universal FCSs in Library Science. A fundamental of such principles is introduced as follows: "The Principles of Homogeneity and Mutual Exclusivity state respectively that facets must be homogeneous and mutually exclusive, i.e., that the contents of any two facets cannot overlap, and that each facet must represent only one characteristic of division of the parent universe".

In this sense, each facet can be designed separately and it models the domain of discourse from a distinct aspect. Each facet consists of a terminology, a finite set of terms that exhaust the facet. This set of terms is also referred to as foci.

There are numerous types of FCSs that vary in complexity. For example, FCSs that include several subject fields containing multiple facets and subfacets

${ }_{2}^{1}$ http://www.rkbexplorer.com/

2 http://www.resist-noe.org/ 
[11] ( $\S 8$, Fig. 1). However, the rest of this section characterizes the elements of a simple generic FCS that this paper will refer to hereafter.

\subsection{Structure and Elements}

Definition 1. Elements of a simple generic Faceted Classification Scheme:

- Target Domain Concept (TDC).

- Facets: Facet1, Facet2, ..., rest of facets.

- Terms or foci (organized by facets):

- Facet1: F1Term1, F1Term2, ..., rest of terms in Facet1.

- Facet2: F2Term1, F2Term2, ..., rest of terms in Facet2.

- ... rest of terms by facet.

- Set of items (from the TDC) to classify: Item1, Item2, ..., rest of items.

The following notation is introduced to refer to the elements of a generic FCS in Def. 1

- TDC denotes the domain or universe of discourse. The domain-specific concept targeted by the FCS.

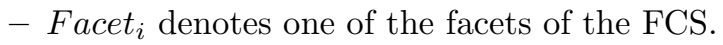

- $F_{i}$ Term $_{j}$ denotes one of the terms of Facet . $_{\text {. }}$.

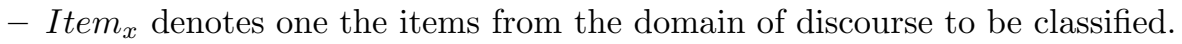

Example 1. The structure below recaps the final FCS developed for the "Dishwashing Detergent" domain example in 8$](\S 2.4)$. The elements of the schema fit into the generic structure presented in Def. 1.

- The $T D C$ element is populated with the domain "Dishwashing Detergent".

- Facet ${ }_{i}$ elements are populated with the facets: "Agent", "Form", "Brand Name", "Scent", "Effect On Agent", and "Special Property".

- $F_{i}$ Term $_{j}$ elements are populated with the terms or foci listed below (grouped by facet):

- Agent: dishwasher, person.

- Form: gel, gelpac, liquid, powder, tablet.

- Brand Name: Cascade, [...], Palmolive, President's Choice, Sunlight.

- etc.

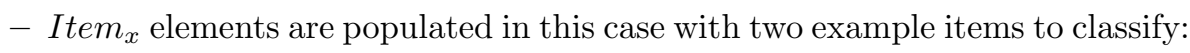

- "President's Choice Antibacterial Hand Soap and Dishwashing Liquid".

- "Palmolive Aroma Therapy, Lavender and Ylang Ylang". 


\section{Normalisation Ontology Design Pattern}

This section highlights the main characteristics of the Normalisation ODP relevant to the comparative analysis to a FCS.

The Normalisation pattern is classified as a "Good Practice" ODP in the catalog of ODPs introduced in 6] 7] (available online 3). It can be applied to any OWL DL ontology that consists of a polyhierarchy where some semantic axes can be pointed. Each of those axes will be a module. One of their most powerful features, is the ability of logical reasoners to link these independent ontology modules to allow them to be separately maintained, extended, and re-used.

The pattern also establishes a series of requirements that a normalised ontology should meet, some of which are summarized below:

- The essence for the normalisation proposal is that the primitive skeleton of the domain ontology should consist of disjoint homogeneous trees (also referred to as modules) [5].

- Each primitive class that is part of the primitive skeleton should only have a primitive parent, and primitive sibling classes should be disjoint, creating the modules [6] (§ 4.3.2.1).

- This implies that for any two primitive concepts either one subsumes the other or they are disjoint. Assertion of multiple inheritance relations among primitive concepts are not allowed [5].

- Normalisation allows exactly one unlabelled flavour of is-kind-of link corresponding to the links declared in the primitive skeleton. All others are inferred by the reasoner [5].

\subsection{Structure and Elements}

There are several examples of the generic structure of the Normalisation ODP in the literature 6$](\S 4.3 .2 .1),\left[7\left(\S 6.5 .1, \S\right.\right.$ A.13) and online ${ }^{3}$. Figure 1 presents the specific version of the generic structure that this paper will refer to hereafter, which preserves the required characteristics of the pattern. Every node of the owl:Thing tree in Fig. 1, denotes an owl:Class. The symbol "(三)" indicates that the corresponding node is a defined class. Otherwise, the node is a primitive class. Every node of the owl:topObjectProperty tree denotes an owl:ObjectProperty. Figure 2 depicts a further generalization of the structure in Fig. 1 and introduces the following notation:

- :TDC denotes a primitive class representing the domain concept being normalised.

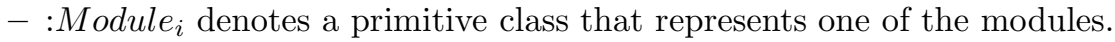

- $: M_{i}$ Class $_{j}$ denotes a primitive class that represents a subset of the module class : Module . $_{\text {. }}$

3 http://odps.sourceforge.net/( $\S$ Normalisation). 


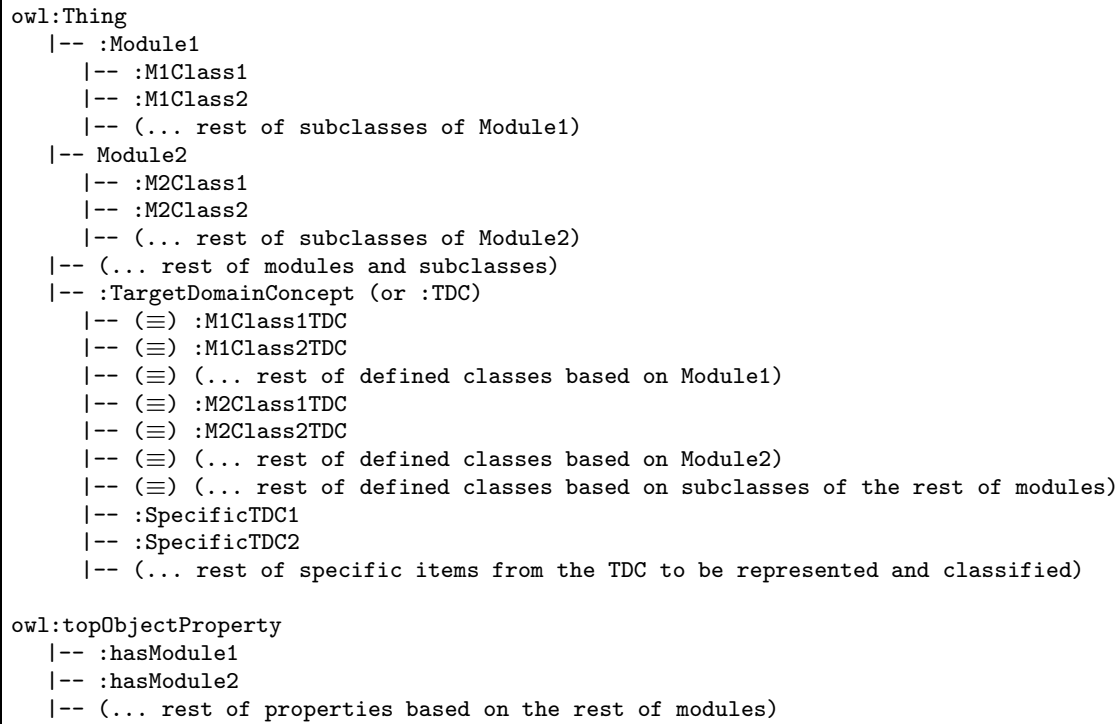

Fig. 1. Generic structure of the Normalisation ODP

- :hasModule denotes an object property that links every module :Module $_{i}$ to the different subclasses of the target domain concept $: M_{i} C l a s s_{j} T D C$ and :SpecificTDC $C_{x}$.

- : $M_{i}$ Class $_{j} T D C$ denotes a defined class that represents a subset of the target domain concept class :TDC. Every class : $M_{i} \mathrm{Class}_{j} T D C$ is defined based on a one-to-one relationship to the single corresponding class : $M_{i} \mathrm{Class}_{j}$ that it is derived from.

- :SpecificTDC $C_{x}$ denotes a primitive class that represents a subset of the target domain concept class :TDC and an entity from the domain to be classified. Every class :SpecificT $D C_{x}$ is described based on a one-to-many relationship to various classes : $M_{i}$ Class $_{j}$ from potentially different modules. As a consequence of this one-to-many relationship, the classes :SpecificT $D C_{x}$ could introduce the polyhierarchy scenarios in the ontology model that the Normalisation ODP aims to manage.

\subsection{Implementation}

One of the main features of the Normalisation ODP is to enable a reasoner to mantain the subsumption relations between a class :SpecificTDC $C_{x}$ and the various classes : $M_{i}$ Class $_{j} T D C$ involved in its description. This feature is accomplished encoding the conditions of the subsumption relation as restrictions in the implementation of the classes : $M_{i}$ Class $_{j} T D C$ and :SpecificT DC $C_{x}$.

Definition 2. The implementation of a generic defined class $: M_{i} \mathrm{Class}_{j} T D C$ is given as follows: 


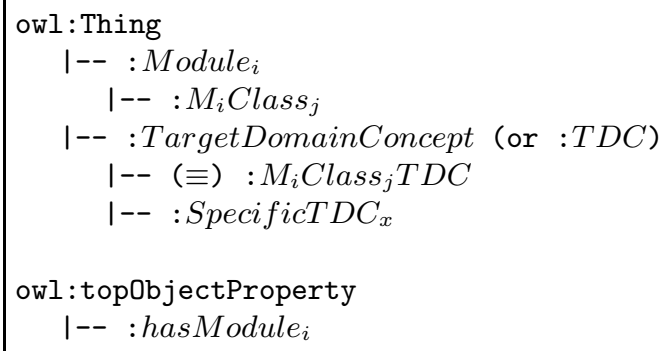

Fig. 2. Generic structure of the Normalisation ODP

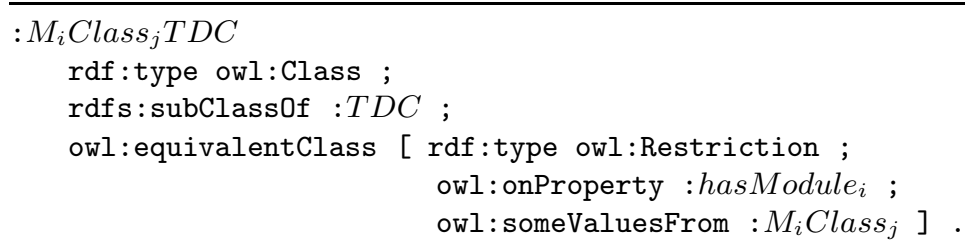

This implementation indicates that:

- A : $M_{i}$ Class $_{j} T D C$ class is equivalent to an anonymous class described by an existential property restriction.

- The restriction is on the object property :hasModule ${ }_{i}$ associated to the module : Module $_{i}$ that subsumes the class : $M_{i}$ Class $_{j}$.

- The filler of the restriction is the class : $M_{i}$ Class $_{j}$ linked to the definition of : $M_{i}$ Class $_{j} T D C$.

Definition 3. The implementation of a generic class :SpecificT $D C_{x}$ is given as follows:

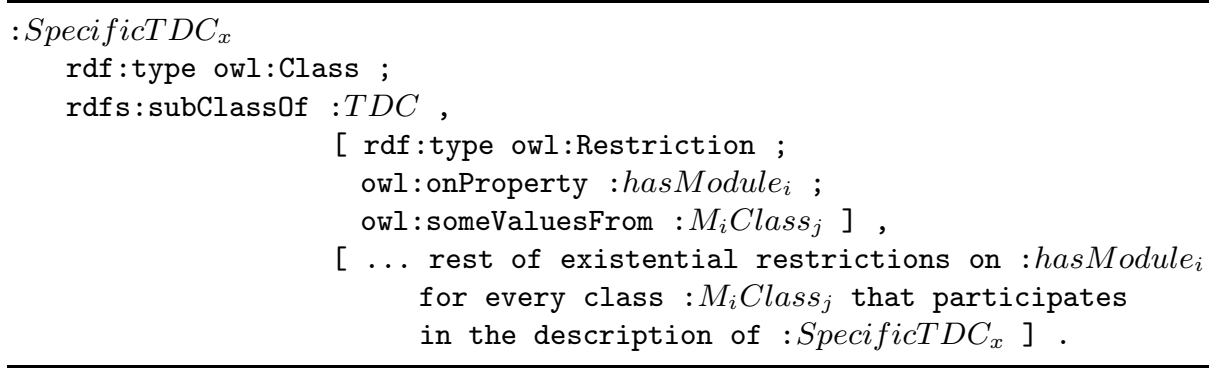

This representation indicates the following:

- A class :SpecificTDC $C_{x}$ is subsumed by a variable number of anonymous classes. More specifically, one anonymous class for every class : $M_{i} \mathrm{Class}_{j}$ of every module : Module $_{i}$ that is linked to the description of :SpecificTDC $C_{x}$. Every anonymous class is represented by an existential property restriction such as: 


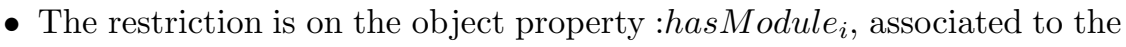
module : Module $_{i}$ that subsumes the class : $M_{i}$ Class $_{j}$.

- The filler of the restriction is the class : $M_{i}$ Class $_{j}$, linked to the description of :SpecificT $D C_{x}$.

This implementation of the classes : $M_{i}$ Class $_{j} T D C$ and :SpecificTDC $C_{x}$ respectively, enable a reasoner to infer and maintain the subsumption relations between a given class :SpecificTDC $C_{x}$ and the various classes : $M_{i} C l a s_{j} T D C$ that it is related to.

Specific examples of the Normalisation ODP in the literature [6] $(\S 4.3 .2 .1)$, [7] $(\S 6.5 .1, \S$ A.13) and online 3 demonstrate the features of the pattern in specific use case scenarios.

\section{Alignment of a FCS to the Normalisation ODP}

A comparative analysis between the main characteristics of a FCS and the Normalisation ODP presented in previous sections, indicates the existence of key similarities between the elements in the generic structures of both conceptual models.

One such key similarity lies in the notion of facet in FCSs and the notion of module (or semantic axis) in the Normalisation ODP. Both elements represent one perspective of the domain being modelled, a single characteristic of division, a single criterion of classification in their respective paradigm.

Another key similarity is linked to the requirement for facets in a FCS to be homogeneous and mutually exclusive and likewise the requirement of modules in the Normalisation ODP to be comprised of primitive classes arranged in a structure of disjoint homogeneous class trees.

These key similarities prompt us to identify a mapping between the elements of both conceptual models that allows to transform a FCS into a normalised ontology model. In this first approach, the mapping aims to keep the design choices of the resultant normalised ontology as simple and straight-forward as possible, without compromising any of the requirements and features of both FCSs and the normalisation mechanism. This approach might not be suitable for converting all possible schemas into a normalised ontology but it is an attempt to provide an initial set of basic design guidelines. These guidelines can be extended hereafter to support more complex cases of FCSs.

The main principle is to represent each facet as a independent module or semantic axis. Following this principle makes the application of the Normalisation ODP almost straight-forward. Moreover, the resultant ontology includes the representation of the multiple alternative classification criteria that were considered in the original FCS for the target domain concept.

Table 1 summarizes the alignment of the elements in the generic structure of both conceptual models. This alignment enables the conversion from a FCS to an OWL DL ontology by applying the Normalisation ODP.

- The first column (leftmost), contains the elements of a generic FCS as introduced in Sect. 2.1, Def. 1. 
Table 1. Alignment of a Faceted Classification Scheme to the Normalisation ODP

\begin{tabular}{|c|c|c|c|}
\hline Library Science & \multicolumn{3}{|c|}{ Ontology Modeling } \\
\hline FCS & Norm. ODP & FCS in Norm. ODP & OWL Implementation \\
\hline$T D C$ & & $: T D C$ & owl:Class (primitive) \\
\hline \multirow{2}{*}{ Facet $_{i}$} & $:$ Module $_{i}$ & $:$ Facet $_{i}$ & owl:Class (primitive) \\
\hline & :hasModule $_{i}$ & :hasFacet ${ }_{i}$ & owl:ObjectProperty \\
\hline \multirow{2}{*}{$F_{i}$ Term $_{j}$} & $: M_{i}$ Class $_{j}$ & $: F_{i}$ Term $_{j}$ & owl:Class (primitive) \\
\hline & $: M_{i}$ Class $_{j} T D C$ & $: F_{i} \operatorname{Term}_{j} T D C$ & owl:Class (defined) $(\equiv)$ \\
\hline Item $_{x}$ & \multicolumn{2}{|c|}{$\overline{: S p e c i f i c T D C_{x}}$} & owl:Class (primitive) \\
\hline
\end{tabular}

- The second column contains the elements of the Normalisation ODP generic structure as introduced in Sect. 3.1, Fig. 2,

- The third column represents the selected OWL notation for the elements of a generic FCS in the context of the Normalisation ODP generic structure.

- The forth column (rightmost), indicates the OWL implementation chosen for every element. The selection complies with the requirements of the normalisation mechanism.

Based on the principle of representating each facet as a module, the underlying ideas behind the mappings in Table 1 can be outlined as follows:

- The target domain concept TDC represents the domain of discourse of both a FCS and the Normalisation ODP. The primitive class :TDC fulfills that role in the normalised ontology.

- A facet Facet ${ }_{i}$ from a generic FCS corresponds to a module : Module $_{i}$ in the Normalisation ODP, therefore it becomes a primitive class : Facet $_{i}$ in the normalised ontology model.

- A facet Facet $_{i}$ from a FCS also becomes an object property :hasFacet in the normalised ontology, given that for every module : Module $_{i}$ in the Normalisation ODP, there is an object property :has Module . $_{\text {. }}$

- From the relationship between facet and module, it follows that a facet term $F_{i}$ Term $_{j}$ from a FCS maps to a module subclass : $M_{i}$ Class $_{j}$ from the Normalisation ODP. Both elements represents the same notion in their respective conceptual models. A subvidision, a refinement of the facet or module that they complement respectively. Therefore, a facet term $F_{i}$ Term ${ }_{j}$ from a FCS becomes a primitive class : $F_{i}$ Term $_{j}$ in the normalised ontology.

- A facet term $F_{i}$ Term $_{j}$ from a FCS also produces a defined class : $F_{i}$ Term $_{j} T D C$ in the normalised ontology, given that for every primitive class : $M_{i}$ Class $_{j}$ in the Normalisation ODP, there is a corresponding defined class : $M_{i} C l a s s_{j} T D C$.

- Every item Item $_{x}$ to be classified in the FCS aligns to a class :Specific $c_{x}$ T DC that is automatically classified by a reasoner in the Normalization ODP. Therefore, every element Item $_{x}$ is represented as a primitive class : SpecificT $D C_{x}$ in the normalised ontology.

The rest of this section details the characteristics of the resultant normalised ontology model that is obtained by applying the Normalisation ODP to a generic 


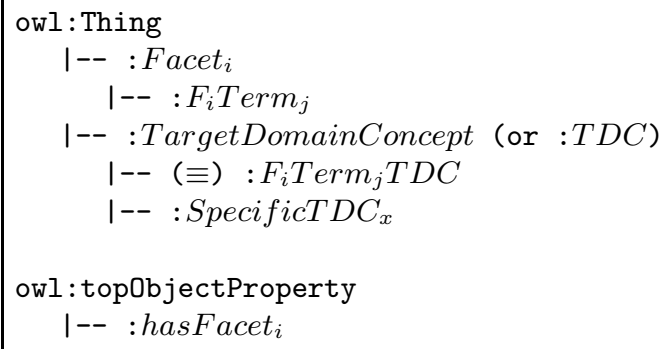

Fig. 3. Elements of a FCS placed into the Normalisation ODP generic structure

FCS. The application of the pattern is driven by the alignments summarized in Table 1. The process is illustrated using the example of the "Dishwashing Detergent" FCS presented in Sect. 2.1, Ex. 1.

\subsection{Structure and Elements}

Figure 3 depicts the placement of the elements of a generic FCS into the generic structure of the Normalisation ODP based on the structure of the pattern in Sect. 3.1. Fig. 2 and the corresponding mappings from Table 1.

Example 2. Now let us populate the generic ontology structure in Fig. 3 with the specific elements of the "Dishwashing Detergent" FCS example. Figure 4 presents the overall normalised ontology class diagram obtained.

It is important to note that the structure in Fig. 4 includes axioms to comply with the requirement already stated of the Normalization ODP. That is, the skeleton of primitive classes consists of disjoint homogeneous tress where each primitive class only has a primitive parent, and primitive sibling classes are disjoint, creating the modules. This normalization requirement complies as well with the FCS requirement of facets being homogeneous and mutually exclusive based on the alignments in Table 1.

\subsection{Implementation}

Defined Classes. The generic implementation of a defined class : $F_{i} T e r m_{j} T D C$ in terms of FCS elements is straight-forward based on the definition of $: M_{i} C_{\text {Class }} T$ TC given in Sect. 3.2, Def. 2 and Table 1.

Example 3. Let us illustrate the implementation of a defined class in the "Dishwashing Detergent" FCS example. Consider the facet "Agent" which contains the terms "Person" and "Dishwasher". From Table 1, these FCS elements fit into the normalised ontology as follows:

- :Facet F $_{i}$ is populated with :Agent. 


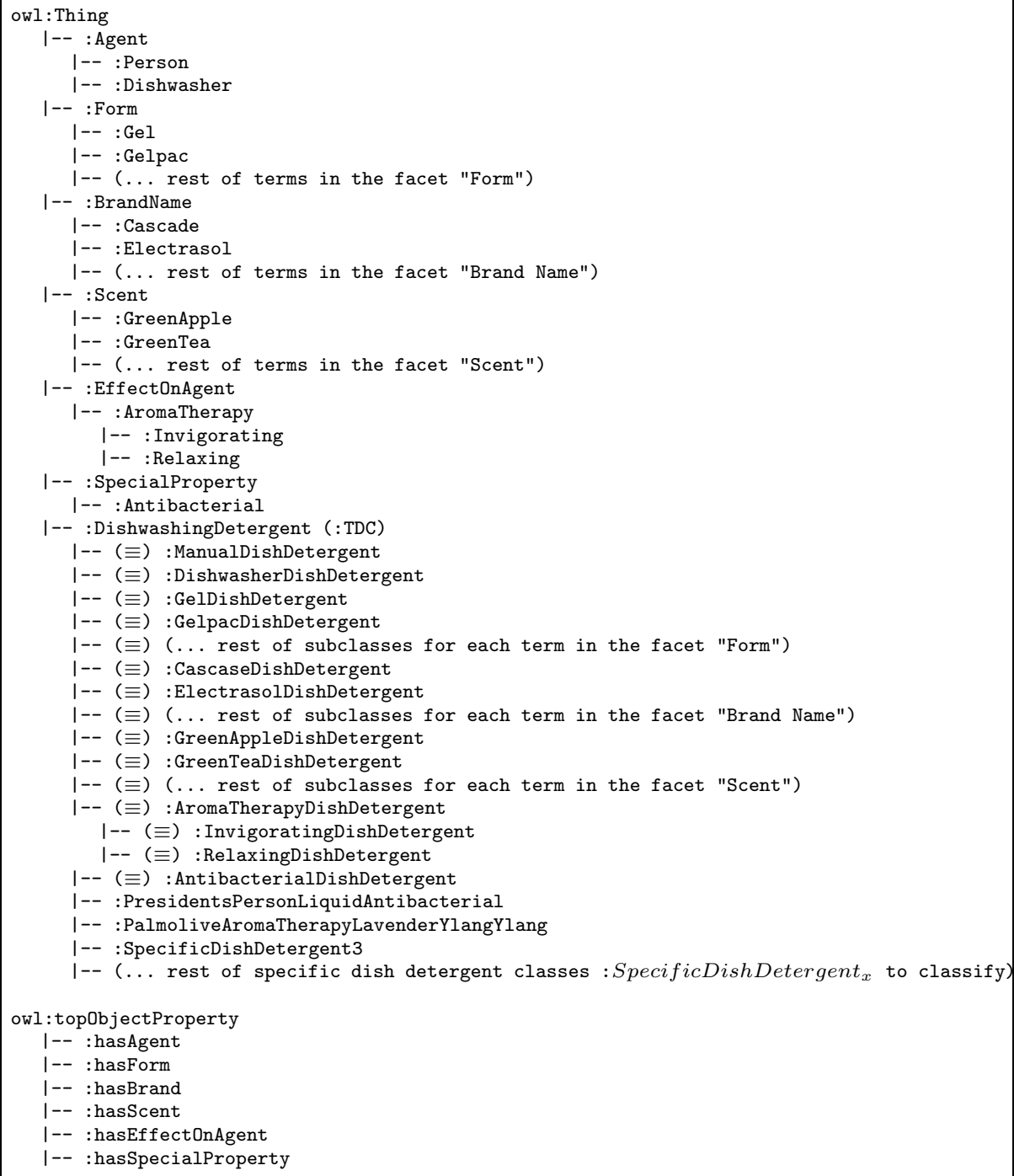

Fig. 4. Normalised ontology structure of the "Dishwashing Detergent" FCS

- :hasFacet ${ }_{i}$ is populated with :hasAgent.

- : $F_{i}$ Term $_{j}$ is populated with :Person and :Dishwasher respectively.

- : $F_{i}$ Term $_{j} T D C$ is populated with :ManualDishDetergent and :DishwasherDishDetergent respectively.

As an example, let us focus on the class :DishwasherDishDetergent. The implementation in the normalised ontology can be stated as follows: 


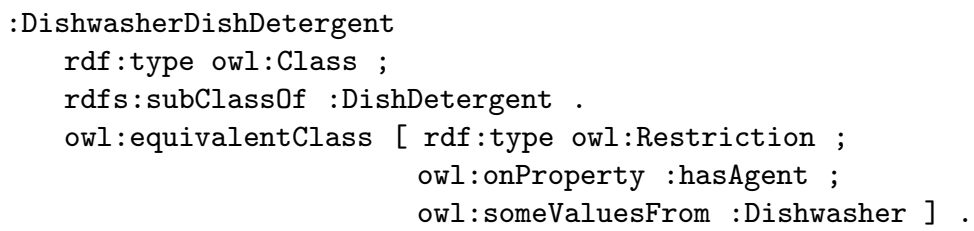

The implementation of the rest defined classes in the "Dishwashing Detergent" FCS shown in Fig. 4 follows the same rationale.

Classification Classes. The generic implementation of a class :SpecificT $D C_{x}$ in terms of FCS elements is straight-forward following the implementation of :SpecificT DC $C_{x}$ given in Sect. 3.2, Def. 3 and Table 1.

Example 4. To illustrate the representation of a specific dishwashing detergent, let us reuse one of the classification examples presented in 8 ( $(\S 2.4)$. The item "President's Choice Antibacterial Hand Soap and Dishwashing Liquid" is classified in the cited reference, as follows: (Agent: person), (Form: liquid), (Brand Name: President's Choice), (Scent: none), (Effect on Agent: none) and (Special Property: antibacterial). From Table1, the description of the example detergent reveals the following mappings:

- :TDC is populated by :DishDetergent.

- :SpecificT $D C_{x}$ is populated by :PresidentsPersonLiquidAntibacterial.

- There are four existential restrictions. One per facet term involved in the description of the specific detergent at hand ("person", "liquid", "President's Choice", and "antibacterial"). Therefore, for each restriction:

- : hasFacet $_{i}$ is populated with :hasAgent, :hasForm, :hasBrandName and :hasSpecialProperty respectively.

- : $F_{i}$ Term $_{j}$ is populated with :Person, :Liquid, :PresidentsChoice and :Antibacterial respectively.

The implementation of this particular detergent in the normalised ontology can be stated as follows:

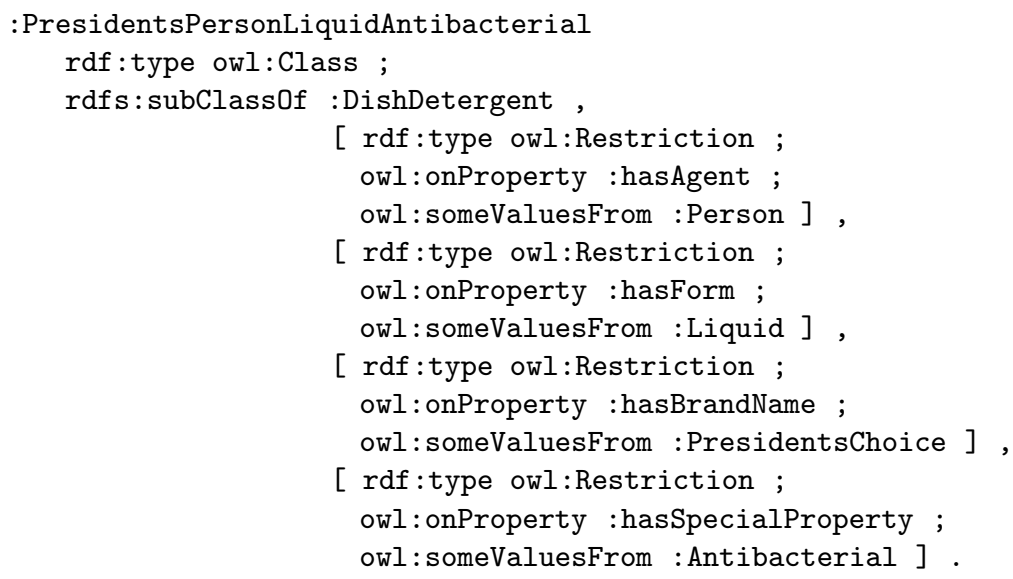


This description makes explicit the relationship between the specific detergent class and every term of every facet that participate in the facet classification of the item. Moreover, it enables a reasoner to infer that :PresidentsPersonLiquidAntibacterial is a subclass of the defined classes :ManualDishDetergent, :LiquidDishDetergent, :PresidentsChoiceDishDetergent and :AntibacterialDishDetergent.

A version of the complete normalised ontology model for the "Dishwashing Detergent" FCS example is available online 4 in RDF/XML format.

\section{Relation to Other Methods}

Previous work that defines mappings between different semantic models include 12. The authors performs a rigorous and comprehensive comparative analysis between the primitive elements of three semantic models: the Semantic Web Ontology Language (OWL), the Relational Database Model (RDBM), and the Resource Space Model (RSM). Based on the identified mappings between every two models, a detailed set of criteria is provided to transform one of them to the other. The most relevant to us is the mapping between RSM and OWL because of its similarities with the conversion between a FCS and OWL that we propose here.

The RSM is defined as a semantic model for specifying, organizing and retrieving diverse multimedia resources by classifying their contents according to different partition methods and organizing them according to a multidimensional classification space. A FCS is also a multidimensional classification space and comparing the primitive elements of a FCS and a RSM the following mapping is instantly revealed:

- The domain or universe of discourse of the FCS (the target domain concept) corresponds to the overall resource space, the RS element in the RSM.

- A facet in the FCS corresponds to an axis $X_{i}$ in the RSM.

- A facet term in the FCS corresponds to a coordinate $C_{i}$ in the RSM.

- A facet is covered and exhausted by the set of terms associated to it in a FCS. The same principle holds in a RSM for an axis and the set of coordinates associated to it, $X_{i}=\left\langle C_{i 1}, C_{i 2}, \ldots, C_{i n}\right\rangle$.

- An item to be classified by the FCS corresponds to a point $p$ in the RSM.

These mappings show that a generic FCS can be converted into a RSM, which in turn can be converted into an OWL model using the RSM to OWL mappings in [12. Now there are two possible paths to convert a FCS into an OWL model.

- Path 1: FCS to RSM via mappings above and RSM to OWL via mappings in [12. Let us refer to this OWL model as $O_{1}$.

- Path 2: FCS to OWL via mappings presented in our paper using the Normalization ODP. Let us refer to this OWL model as $\mathrm{O}_{2}$.

${ }^{4}$ http://purl.org/net/project/enakting/ontology/detergent_fcs_norm 
There are important differences between the ontologies $O_{1}$ and $O_{2}$. An important difference is due to the RSM to OWL conversion in 12. RSM describes mainly classification semantics and as the authors explain, this means that there is no semantic loss when converting from RSM to OWL but there might be semantic loss when transforming an OWL model that includes richer semantics into a RSM. This also means that, in terms of W3C standards, the expressivity level of the resultant OWL model $O_{1}$, will be within the RDF Schema or OWL Lite boundary.

On the other hand, the ontology $\mathrm{O}_{2}$ is within OWL DL and presents richer OWL semantics than $O_{1}$, provided by the Normalization ODP. These additional OWL DL semantics in $\mathrm{O}_{2}$ enable one of the main features of the normalization pattern such as the automatic classification and maintenance of complex subsumption relations by a reasoner. So while $O_{1}$ is a valid OWL description of the FCS that it is based on, $\mathrm{O}_{2}$ using our proposed method provides additional semantics at the OWL DL level that support a richer description and additional features of the classification criteria considered in the initial FCS.

Additional research that made use of facet analysis in Library and Information Science to build computational ontologies includes [13. Giunchiglia et al. introduces the concept of Faceted Lightweight Classification Ontology as "a lightweight (classification) ontology where each term and corresponding concept occurring in its node labels must correspond to a term and corresponding concept in the background knowledge, modeled as a faceted classification scheme".

Similarities to our approach include:

- The use of a FCS to model certain background knowledge and to derive and ontology based on it.

- Each concept in the ontology model obtained in our method also corresponds to a concept in the FCS.

There are important differences where our approach deviates from that in 13 . probably due to the different type of problems that both are trying to address respectively. Giunchiglia et al. are trying to counteract the lack of interest and difficulties on the user side to build and reuse ontologies while our concern focuses on identifying explicit guidelines to represent the notion of multiple classification criteria in domain concepts. Additional differences include:

- The expressive level for the resultant ontology model in our method is OWL DL. In contrast, [13] focuses on lightweight classification ontologies which expressive level would loosely correspond to no more than RDF Schema in terms of W3C Standards. Key features provided by the Normalisation ODP found in our method, can not be implemented using solely RDF Schema semantics.

- The type of FCS used in [13] is based on a Universal Faceted Classification System. On the other hand, we have focused on simpler custom domainspecific FCSs to serve as an starting point for our initial proof of concept. This helped limiting the complexity of the classification criteria to consider and represent in the corresponding ontology. 


\section{Conclusions}

This paper has presented an initial set of basic design guidelines to develop an ontology model within OWL DL that supports the representation of multiple alternative classification criteria of a specific domain concept.

A lack of explicit guidance in the ontology development literature on how to address this recurrent modeling scenario, leaves ample room for ad-hoc practices that can lead to unexpected or undesired results in ontology artifacts. In our attempt to mitigate this void, we examined a simplified procedure to develop a Faceted Classification Scheme (FCS) which contains the conceptualization of various classification criteria (facets) of a specific target domain concept. A series of mappings between the elements of a generic FCS and the Normalization Ontology Design Pattern (ODP) have been identified that allow us to convert a given FCS into an OWL DL ontology model following a consistent and systematic approach. The resultant ontology model includes the representation of the various classification criteria of the domain concept considered in the original FCS. An existing FCS example in the domain of "Dishwashing Detergent" is used to illustrate the main steps of our conversion procedure.

The guidelines presented in this first effort consider explicitly the conceptualization of existing classification criteria in the context of ontology modeling for the Semantic Web and provide a partial solution to the problem scenario described. They do not cover all existing types of generic structures of FCSs (which can be the aim of future work) and they do not eliminate all opportunities of potentially hazardous ad-hoc decisions in the development process. However, we believe the use of a consistent, systematic and fit-for-purpose approach allows to significantly reduced them.

Acknowledgments. This work was supported in part by the UK Engineering and Physical Sciences Research Council (EPSRC), in the context of the EnAKTing project under grant number EP/G008493/1. Additionally, many people have contributed directly and indirectly to this work and we thank them all. In particular, everyone that participated in the online discussion "The notion of a 'classification criterion' as a class" [5 via the mailing lists: "ontologforum@ontolog.cim3.net" and "public-owl-dev@w3c.org"; and the British Chapter of the International Society for Knowledge Organization (ISKO).

\section{References}

1. Welty, C., McGuinness, D.L., Smith, M.K.: OWL web ontology language guide. W3C recommendation, W3C (February 2004), http://www .w3.org/TR/2004/REC-owl-guide-20040210/

2. Krötzsch, M., Patel-Schneider, P.F., Rudolph, S., Hitzler, P., Parsia, B.: OWL 2 web ontology language primer. Technical report, W3C (October 2009), http://www.w3.org/TR/2009/REC-ow12-primer-20091027/

\footnotetext{
${ }^{5}$ http://lists.w3.org/Archives/Public/public-owl-dev/2010AprJun/0009.html

6 http://ontolog.cim3.net/forum/ontolog-forum/2010-04/msg00051.html
} 
3. Horridge, M., Drummond, N., Jupp, S., Moulton, G., Stevens, R.: A practical guide to building owl ontologies using the protege-owl plugin and co-ode tools edition 1.2. Technical report, The University Of Manchester (March 2009)

4. Spiteri, L.: A simplified model for facet analysis: Ranganathan 101. Canadian Journal of Information and Library Science 23(1/2), 1-30 (1998)

5. Rector, A.L.: Modularisation of domain ontologies implemented in description logics and related formalisms including owl. In: Proceedings of the 2nd International Conference on Knowledge Capture, K-CAP 2003, pp. 121-128. ACM, New York (2003)

6. Egana-Aranguren, M.: Ontology Design Patterns for the Formalisation of Biological Ontologies. MPhil Dissertation, Bio-Health Informatics Group, School of Computer Science, University of Manchester (2005)

7. Egana-Aranguren, M.: Role and Application of Ontology Design Patterns in Bioontologies. PhD thesis, School of Computer Science, University of Manchester (2009)

8. Denton, W.: How to make a faceted classification and put it on the web (November 2003), http://www.miskatonic.org/library/facet-web-howto.html

9. Rodriguez-Castro, B., Glaser, H.: Whose "fault" is this? untangling domain concepts in ontology design patterns. In: Workshop on Knowledge Reuse and Reengineering over the Semantic Web in the 5th European Semantic Web Conference (June 2008)

10. Kwasnik, B.H.: The role of classification in knowledge representation and discovery. Library Trends 48(1) (1999)

11. Vickery, B.: Faceted classification for the web. Axiomathes 18(2), 145-160 (2008)

12. Zhuge, H., Xing, Y., Shi, P.: Resource space model, owl and database: Mapping and integration. ACM Trans. Internet Technol. 8(4), 1-31 (2008)

13. Giunchiglia, F., Dutta, B., Maltese, V.: Faceted lightweight ontologies. In: Borgida, A., Chaudhri, V.K., Giorgini, P., Yu, E.S.K. (eds.) Conceptual Modeling: Foundations and Applications. LNCS, vol. 5600, pp. 36-51. Springer, Heidelberg (2009) 